\title{
On Solving Linear Fractional Programming Problems
}

\author{
P. Pandian ${ }^{1} \&$ M. Jayalakshmi ${ }^{1}$ \\ ${ }^{1}$ Department of Mathematics, School of Advanced Sciences, VIT University, Vellore, India \\ Correspondence: P. Pandian, Department of Mathematics, School of Advanced Sciences, VIT University, \\ Vellore 632014, India. E-mail: pandian61@rediffmail.com
}

Received: March 14, 2013

doi:10.5539/mas.v7n6p90
Accepted: April 18, 2013 Online Published: May 24, 2013

URL: http://dx.doi.org/10.5539/mas.v7n6p90

\begin{abstract}
A new method namely, denominator objective restriction method based on simplex method is proposed for solving linear fractional programming problems. Further, another method namely, decomposition-restriction method based on decomposition principle and the denominator objective restriction method is proposed for obtaining an optimal fuzzy solution to the fully fuzzy linear fractional programming problem. The procedures for the proposed methods are illustrated with the numerical examples.
\end{abstract}

Keywords: linear fractional programming, optimal solution, denominator objective restriction method, fuzzy numbers, fully fuzzy linear fractional programming

\section{Introduction}

Linear fractional programming (LFP) problems are a special type of non-linear programming problems in which the objective function is a ratio of linear functions and the constraints are linear functions. In real life situations, linear fractional models arise in decision making such as construction planning, economic and commercial planning, health care and hospital planning. In the literature, several methods (Bajalinov, 2003; Stancu-Minasian, 1997, 2006) have been recommended to solve LFP problems. Isbell and Marlow (1956) first identified an example of LFP problem and solved it by a sequence of linear programming problems. Charnes and Cooper (1962) considered variable transformation method to solve LFP and the updated objective function method were developed for solving the LFP problem by Bitran and Novaes (1973). Gilmore and Gomory (1963), Martos (1964), Swarup (1965), Wagner and Yuan (1968), Pandey and Punnen (2007) and Sharma et al. (1980) solved the LFP problem by various types of solution procedures based on the simplex method developed by Dantzig (1962). Tantawy $(2007,2008)$ proposed two different approaches namely; a feasible direction approach and a duality approach to solve the LFP problem. Mojtaba Borza et al. (2012) solved the LFP problem with interval coefficients in objective function which is based on Charnes and Cooper technique (1962). Odior (2012) solved the LFP problem by algebraic approach which depends on the duality concept and the partial fractions.

In real life model, the possible values of coefficients of a linear programming problem are obviously unclear and vague. In fuzzy decision making problems, the idea of maximizing decision was anticipated by Bellman and Zadeh (1970). The theory of fuzzy linear programming on general level was initially proposed by Tanaka et al. (1973). Buckley and Feuring (2000) measured the fully fuzzified linear programming problem (FFLP) by transformation to multiple objective deterministic non-linear programming problems. Li and Chen (1996) solved a fuzzy linear fractional programming form by fuzzy coefficients, using the concept as well as mathematical definition of the fuzzy optimal. Jayalakshmi and Pandian (2012) have proposed a method namely, bound and decomposition method to a fully fuzzy linear programming (FFLP) problem to obtain an optimal fuzzy solution. Hashemi et al. (2006) planned a two-phase approach based on the evaluation of mean plus standard deviation of fuzzy numbers to get the optimal solutions of the FFLP problem. Mikaeilvand et al. (2008) projected a method to solve FFLP through defuzzifying with a linear ranking function. Pop and Stancu Minasian (2008) and Bogdana Stanojevi'ca and Stancu-Minasianb (2012) used deterministic multiple objective linear programming problem by quadratic constraints to work out FFLP problems. Nachammai et al. (2012) considered FFLFP problem by using ranking method based on metric distance.

In this paper, we propose a new method namely, denominator objective restriction method for finding an optimal solution to LFP problems. In this proposed method, we construct two linear programming problems from the given LFP problem such that one is of maximization type and the other is of minimization type. Then, we attain 
an optimal solution to the given LFP problem from the solutions of the two constructed linear programming problems. The proposed method is based only on the simplex method which differs totally from transformation method introduced by Charnes and Copper (1962) and the fractional simplex method introduced by Swarup (1965). Further, based on the decomposition principle and the denominator objective restriction method, we develop a new method namely, decomposition-restriction method to the FFLFP problem. In the decomposition-restriction method, the fuzzy ranking function, the transformation technique and multi-objective non-linear programming technique are not used. Numerical examples are given for better understanding the solution procedures of the proposed methods.

\section{Preliminaries}

We require the following definitions of the basic arithmetic operators and partial ordering relations on fuzzy triangular numbers based on the function principle which can be established in Bellman and Zadeh (1970), Jayalakshmi and Pandian (2012) and are used in section 4.

Definition 2.1 A fuzzy number $\tilde{a}$ is a triangular fuzzy number denoted by $\left(a_{1}, a_{2}, a_{3}\right)$ where $a_{1}, a_{2}$ and $a_{3}$ are real numbers and its membership function $\mu_{\tilde{a}}(x)$ is given below:

$$
\mu_{\tilde{a}}(x)=\left\{\begin{array}{cc}
\left(x-a_{1}\right) /\left(a_{2}-a_{1}\right) & \text { for } a_{1} \leq x \leq a_{2} \\
\left(a_{3}-x\right) /\left(a_{3}-a_{2}\right) & \text { for } a_{2} \leq x \leq a_{3} \\
0 & \text { otherwise }
\end{array}\right.
$$

Definition 2.2 Let $\left(a_{1}, a_{2}, a_{3}\right)$ and $\left(b_{1}, b_{2}, b_{3}\right)$ be two triangular fuzzy numbers. Then,

(i) $\left(a_{1}, a_{2}, a_{3}\right) \oplus\left(b_{1}, b_{2}, b_{3}\right)=\left(a_{1}+b_{1}, a_{2}+b_{2}, a_{3}+b_{3}\right)$.

(ii) $\left(a_{1}, a_{2}, a_{3}\right) \Theta\left(b_{1}, b_{2}, b_{3}\right)=\left(a_{1}-b_{3}, a_{2}-b_{2}, a_{3}-b_{1}\right)$.

(iii) $k\left(a_{1}, a_{2}, a_{3}\right)=\left(k a_{1}, k a_{2}, k a_{3}\right)$, for $k \geq 0$.

(iv) $k\left(a_{1}, a_{2}, a_{3}\right)=\left(k a_{3}, k a_{2}, k a_{1}\right)$, for $k<0$.

(v) $\left(a_{1}, a_{2}, a_{3}\right) \otimes\left(b_{1}, b_{2}, b_{3}\right)= \begin{cases}\left(a_{1} b_{1}, a_{2} b_{2}, a_{3} b_{3}\right), & a_{1} \geq 0, \\ \left(a_{1} b_{3}, a_{2} b_{2}, a_{3} b_{3}\right), & a_{1}<0, a_{3} \geq 0, \\ \left(a_{1} b_{3}, a_{2} b_{2}, a_{3} b_{1}\right), & a_{3}<0 .\end{cases}$

(vi) If $0 \notin\left(b_{1}, b_{2}, b_{3}\right), \frac{\left(a_{1}, a_{2}, a_{3}\right)}{\left(b_{1}, b_{2}, b_{3}\right)}=\left(\frac{a_{1}}{b_{3}}, \frac{a_{2}}{b_{2}}, \frac{a_{3}}{b_{1}}\right)$.

Let $F(R)$ be the set of all real triangular fuzzy numbers.

Definition 2.3 Let $\tilde{A}=\left(a_{1}, a_{2}, a_{3}\right)$ and $\tilde{B}=\left(b_{1}, b_{2}, b_{3}\right)$ be in $F(R)$, then

(i) $\widetilde{A} \approx \widetilde{B}$ if $a_{\mathrm{i}}=b_{\mathrm{i}}, i=1,2,3$; (ii) $\tilde{A} \preceq \tilde{B}$ if $a_{\mathrm{i}} \leq b_{\mathrm{i}}, i=1,2,3$

(iii) $\tilde{A} \succeq \tilde{B}$ if $a_{\mathrm{i}} \geq b_{\mathrm{i}}, i=1,2,3$ and $\tilde{A} \succeq \tilde{0}$ if $a_{\mathrm{i}} \geq 0, i=1,2,3$.

\section{Linear Fractional Programming Problems}

Consider the following LFP problem:

(P) Maximize $Z=\frac{C^{T} X+\alpha}{D^{T} X+\beta}$, subject to $A X \leq B, \quad X \geq 0$

where $X, C, D$ are $n \times 1$ vectors, $B$ is an $m \times 1$ vector and $\alpha, \beta$ are scalars.

It is assumed that the set of feasible solutions to the problem (P), $S=\left\{X \in R^{n}: A X \leq b, X \geq 0\right\}$ is non-empty and bounded.

Now, we can construct two single objective linear programming problem from the given problem (P) as follows:

(N) Maximize $P(X)=C^{T} X+\alpha$, subject to $A X \leq B, X \geq 0$

and

(D) Minimize $Q(X)=D^{T} X+\beta$, subject to $A X \leq B, X \geq 0$

Now, we prove the following two theorems connecting the solutions of the problem (P), the problem $(\mathrm{N})$ and the problem (D) which are used in the proposed method. 
Theorem 3.1 Let $X_{\circ}$ be an optimal solution to the problem (N). If $\left\{X_{n}\right\}$ is a sequence of basic feasible solutions to the problem (D) such that $Z\left(X_{k}\right) \leq Z\left(X_{k+1}\right)$ for all $k=0,1,2, \ldots, n-1$ and $Z\left(X_{n}\right) \geq Z\left(X_{n+1}\right)$ by simplex method considering the solution $X_{\circ}$ as an initial feasible solution, then $X_{n}$ is an optimal solution to the problem (P).

Proof: It is obvious that $X_{n}$ is a feasible solution to the problem (P)

Let $\mathrm{U}$ be a feasible solution to the problem $(\mathrm{P})$.

This implies $Q(U) \leq Q\left(X_{n}\right)$ or $Q(U)>Q\left(X_{n}\right)$.

Case 1: $Q(U) \leq Q\left(X_{n}\right)$

Since $Z\left(X_{k}\right) \leq Z\left(X_{k+1}\right)$ for all $k=0,1,2, \ldots, n-1$ and $Z\left(X_{n}\right) \geq Z\left(X_{n+1}\right)$ and the problem (D) is of minimization type, we have $Z\left(X_{n}\right) \geq Z(U)$. Therefore, $X_{n}$ is an optimal solution to the problem (P).

Case 2: $Q(U)>Q\left(X_{n}\right)$. That is, $Q\left(X_{n}\right)<Q(U)$

Since $Z\left(X_{k}\right) \leq Z\left(X_{k+1}\right)$ for all $k=0,1,2, \ldots, n-1$ and $Z\left(X_{n}\right) \geq Z\left(X_{n+1}\right)$ and also, the problem (D) is of minimization type, we can conclude that $Z\left(X_{n}\right) \geq Z(U)$. Therefore, $X_{n}$ is an optimal solution to the problem (P).

Thus, $X_{n}$ is an optimal solution to the problem (P).

Thus, the theorem is proved.

Theorem 3.2 Let $X_{\circ}$ be an optimal solution to the problem (N). If $\left\{X_{n}\right\}$ is a sequence of basic feasible solutions to the problem (D) such that $Z\left(X_{k}\right) \leq Z\left(X_{k+1}\right)$ for all $k=0,1,2, \ldots, n$ and $X_{n+1}$ is an optimal solution to the problem (D) by simplex method considering the solution $X_{\circ}$ as an initial feasible solution, then $X_{n+1}$ is an optimal solution to the problem (P).

Proof: It is observed that $X_{n+1}$ is a feasible solution to the problem (P).

Let $\mathrm{V}$ be a feasible solution to the problem (P).

Now, since $X_{n+1}$ is an optimal solution to the problem (D), we have $Q(V) \geq Q\left(X_{n+1}\right)$.

Now, since $Z\left(X_{k}\right) \leq Z\left(X_{k+1}\right)$ for all $k=0,1,2, \ldots, n$ and $X_{n+1}$ is an optimal solution to the problem (D), we can conclude that $Z\left(X_{n+1}\right) \geq Z(V)$. Therefore, $X_{n+1}$ is an optimal solution to the problem (P).

Hence, the theorem is proved.

Now, we introduce a new method namely, denominator objective restriction method for finding an optimal solution to the LFP problem (P).

The proposed method proceeds as follows:

Step 1: Construct two single objective linear programming problems namely, the problem $(\mathrm{N})$ as well as the problem (D) from the given problem (P).

Step 2: Compute the optimal solution to the problem $(\mathrm{N})$ by means of the simplex method. Let the optimal solution to the problem (N) be $X_{\circ}$ and Max. $Z\left(X_{\circ}\right)=Z$ 。

Step 3: Using the optimal table of the problem $(N)$ as an initial simplex table to the problem (D), continue to find a sequence of improved basic feasible solutions $\left\{X_{n}\right\}$ to the problem (D) and the value of $Z$ at each of the improved basic feasible solution by the simplex method.

Step 4: (a) If $Z\left(X_{k}\right) \leq Z\left(X_{k+1}\right)$ for all $k=0,1,2, \ldots, n-1$ and $Z\left(X_{n}\right) \geq Z\left(X_{n+1}\right)$ for some $\mathrm{n}$, stop the computation process and then, go to Step 5 .

Step 4: (b) If $Z\left(X_{k}\right) \leq Z\left(X_{k+1}\right)$ for all $k=0,1,2, \ldots, n$ and $X_{n+1}$ is an optimal solution to the problem (D) for some $n$, stop the computation process and then, go to Step 6 .

Step 5: $X_{n}$ is an optimal solution to the problem (P) and Max. $Z(X)=Z\left(X_{n}\right)$ by the Theorem 3.1.

Step 6: $X_{n+1}$ is an optimal solution to the problem (P) and Max. $Z(X)=Z\left(X_{n+1}\right)$ by the Theorem 3.2.

Remark 3.1 The maximum value for $(n+1)$ is the number of the iterations to get an optimal solution to the problem (D) using simplex method.

The proposed method for solving the LFP problem is illustrated through the following examples.

Example 3.1 Consider the following LFP problem: 


$$
\text { Maximize } Z=\frac{5 x_{1}+6 x_{2}}{2 x_{2}+7}
$$

subject to $2 x_{1}+3 x_{2} \leq 6 ; 2 x_{1}+x_{2} \leq 3 ; x_{1}, x_{2} \geq 0$.

The following two LP problems can be obtained from the given problem:

(N) Maximize $P(X)=5 x_{1}+6 x_{2}$

subject to $2 x_{1}+3 x_{2} \leq 6 ; 2 x_{1}+x_{2} \leq 3 ; x_{1}, x_{2} \geq 0$

and

(D) Minimize $Q(X)=2 x_{2}+7$

subject to $2 x_{1}+3 x_{2} \leq 6 ; 2 x_{1}+x_{2} \leq 3 ; x_{1}, x_{2} \geq 0$.

Now, the optimal solution to the problem $(\mathrm{N})$, by the simplex method, is given by the following table:

\begin{tabular}{cccccccc}
\hline & $C$ & 5 & 6 & 0 & 0 & & \\
\hline$C_{B}$ & $X_{B}$ & $x_{1}$ & $x_{2}$ & $s_{1}$ & $s_{2}$ & Solution. & Ratio \\
\hline 6 & $x_{2}$ & 0 & 1 & $\frac{1}{2}$ & $-\frac{1}{2}$ & $\frac{3}{2}$ & \\
5 & $x_{1}$ & 1 & 0 & $-\frac{1}{4}$ & $\frac{3}{4}$ & $\frac{3}{4}$ & \\
\hline$P_{j}-C_{j}$ & 0 & 0 & $\frac{7}{4}$ & $\frac{3}{4}$ & $P=\frac{51}{4}$ & $Z=\frac{51}{40}$
\end{tabular}

Therefore, the optimal solution to the problem $(\mathrm{N})$ is $x_{1}=\frac{3}{4}, x_{2}=\frac{3}{2}$, max. $P(X)=\frac{51}{4}$ and the value of $Z=\frac{51}{40}$.

Now, by Step 3 of the proposed method, the initial simplex table to the problem (D) is given below:

\begin{tabular}{cccccccc}
\hline & $D$ & 0 & 2 & 0 & 0 & & \\
\hline$D_{B}$ & $X_{B}$ & $x_{1}$ & $x_{2}$ & $S_{1}$ & $s_{2}$ & Solution. & Ratio \\
\hline 2 & $x_{2}$ & 0 & 1 & $\frac{1}{2}$ & $-\frac{1}{2}$ & $\frac{3}{2}$ & - \\
0 & $x_{1}$ & 1 & 0 & $-\frac{1}{4}$ & $\frac{3}{4}$ & $\frac{3}{4}$ & 1 \\
\hline$D_{j}-Q_{j}$ & 0 & 0 & -1 & 1 & $Q=10$ & $Z_{\circ}=\frac{51}{40}$ \\
\hline
\end{tabular}

Now, the variable $s_{2}$ enters into the basis and the variable $x_{1}$ leaves from the basis.

\section{Ist iteration table:}

\begin{tabular}{cccccccc}
\hline & $D$ & 0 & 2 & 0 & 0 & & \\
\hline$D_{B}$ & $X_{B}$ & $x_{1}$ & $x_{2}$ & $s_{1}$ & $s_{2}$ & Solution. & Ratio \\
\hline 0 & $S_{1}$ & 0 & 2 & 1 & -1 & 3 & \\
0 & $x_{1}$ & 1 & $\frac{1}{2}$ & 0 & $\frac{1}{2}$ & $\frac{3}{2}$ & \\
\hline \multicolumn{2}{l}{$D_{j}-Q_{j}$} & 0 & 0 & 0 & 0 & $Q=7$ & $Z_{1}=\frac{15}{14}$ \\
\hline
\end{tabular}


Since $Z_{\circ}>Z_{1}$ and by the Step 4(a) of the proposed method, the optimal solution to the given linear fractional programming problem is $x_{1}=\frac{3}{4}, x_{2}=\frac{3}{2}$ and Max. $Z=\frac{51}{40}$.

Example 3.2 Consider the following LFP problem:

Maximize $Z=\frac{x_{1}+2 x_{2}}{2 x_{1}-x_{2}+2}$

subject to $-x_{1}+2 x_{2} \leq 2 ; x_{1}+x_{2} \leq 4 ; x_{1}, x_{2} \geq 0$.

The following two LP problems can be obtained from the given problem:

(N) Maximize $P(X)=x_{1}+2 x_{2}$

subject to $-x_{1}+2 x_{2} \leq 2 ; x_{1}+x_{2} \leq 4 ; x_{1}, x_{2} \geq 0$.

and

(D) Minimize $Q(X)=2 x_{1}-x_{2}+2$

subject to $-x_{1}+2 x_{2} \leq 2 ; x_{1}+x_{2} \leq 4 ; x_{1}, x_{2} \geq 0$.

Now, by the simplex method, the optimal solution to the problem $(\mathrm{N})$ is given in the following table:

\begin{tabular}{cccccccc}
\hline & $C$ & 1 & 2 & 0 & 0 & & \\
\hline$C_{B}$ & $X_{B}$ & $x_{1}$ & $x_{2}$ & $s_{1}$ & $s_{2}$ & Solution. & Ratio \\
\hline 2 & $x_{2}$ & 0 & 1 & $\frac{1}{3}$ & $\frac{1}{3}$ & 2 & \\
1 & $x_{1}$ & 1 & 0 & $-\frac{1}{3}$ & $\frac{2}{3}$ & 2 & \\
\hline$P_{j}-C_{j}$ & 0 & 0 & $\frac{1}{3}$ & $\frac{4}{3}$ & $P=6$ & $Z=\frac{3}{2}$ \\
\hline
\end{tabular}

Therefore, the optimal solution to the problem $(\mathrm{N})$ is $x_{1}=2, x_{2}=2$, Max. $P(X)=6$ and the value of $Z=\frac{3}{2}$.

Now, by Step 3 of the proposed method, the initial simplex table to the problem (D) is given below:

\begin{tabular}{cccccccc}
\hline & $D$ & 2 & -1 & 0 & 0 & & \\
\hline$D_{B}$ & $X_{B}$ & $x_{1}$ & $x_{2}$ & $s_{1}$ & $s_{2}$ & Solution. & Ratio \\
\hline-1 & $x_{2}$ & 0 & 1 & $\frac{1}{3}$ & $\frac{1}{3}$ & 2 & 6 \\
2 & $x_{1}$ & 1 & 0 & $-\frac{1}{3}$ & $\frac{2}{3}$ & 2 & 3 \\
\hline$D_{j}-Q_{j}$ & 0 & 0 & 1 & -1 & $Q=4$ & $Z_{\circ}=\frac{3}{2}$
\end{tabular}

Now, the variable $s_{2}$ enters into the basis and the variable $x_{1}$ leaves from the basis. 


\section{Ist iteration table:}

\begin{tabular}{cccccccc}
\hline & $D$ & 2 & -1 & 0 & 0 & & \\
\hline$D_{B}$ & $X_{B}$ & $x_{1}$ & $x_{2}$ & $s_{1}$ & $s_{2}$ & Solution. & Ratio \\
\hline-1 & $x_{2}$ & $-\frac{1}{2}$ & 1 & $\frac{1}{2}$ & 0 & 1 & \\
0 & $S_{2}$ & $\frac{3}{2}$ & 0 & $-\frac{1}{2}$ & 1 & 3 & \\
\hline$D_{j}-Q_{j}$ & 0 & 0 & 0 & 0 & $Q=1$ & $Z_{1}=2$
\end{tabular}

Since the $\overline{\mathrm{I}^{\text {st }}}$ iteration table is optimal and by Step 4(b) of the proposed method, the optimal solution to the given linear fractional programming problem is $x_{1}=0, x_{2}=1$ and Max. $Z=2$.

\section{Fully Fuzzy Linear Fractional Programming Problem}

Consider the following FFLFP problems having $m$ fuzzy constraints and $n$ fuzzy variables:

(FP) Maximize $\tilde{z} \approx \frac{\tilde{c}^{T} \tilde{x} \oplus \tilde{\alpha}}{\tilde{d}^{T} \tilde{x} \oplus \tilde{\beta}}$, subject to $\tilde{A} \otimes \tilde{x}\{\preceq, \approx, \succeq\} \tilde{b}, \quad \tilde{x} \succeq \tilde{0}$

where $\tilde{c}^{T}=\left(\tilde{c}_{j}\right)_{1 \times n}, \quad \tilde{d}^{T}=\left(\tilde{d}_{j}\right)_{1 \times n}, \quad \tilde{A}=\left(\tilde{a}_{i j}\right)_{m \times n}, \quad \tilde{x}=\left(\tilde{x}_{j}\right)_{n \times 1}, \tilde{b}=\left(\tilde{b}_{i}\right)_{m \times 1}, \quad \tilde{\alpha}, \tilde{\beta} \in F(R)$

and $\tilde{a}_{i j}, \tilde{c}_{j}, \tilde{d}_{j}, \tilde{x}_{j}, \tilde{b}_{i} \in F(R)$, for all $1 \leq j \leq n$ and $1 \leq i \leq m$.

Let the parameters $\tilde{\alpha}, \tilde{\beta}, \tilde{c}_{j}, \tilde{d}_{j}, \tilde{x}_{j}, \tilde{a}_{i j}$ and $\tilde{b}_{i}$ be the triangular fuzzy numbers $\left(\alpha_{1}, \alpha_{2}, \alpha_{3}\right),\left(\beta_{1}, \beta_{2}, \beta_{3}\right)$, $\left(p_{j}, q_{j}, r_{j}\right),\left(m_{j}, n_{j}, l_{j}\right),\left(x_{j}, y_{j}, t_{j}\right),\left(a_{i j}, b_{i j}, c_{i j}\right)$ and $\left(b_{i}, g_{i}, h_{i}\right)$ respectively. Then, the problem (FP) can be written as follows:

(FP) Maximize $\left(z_{1}, z_{2}, z_{3}\right) \approx \frac{\left(\sum_{j=1}^{n}\left(p_{j}, q_{j}, r_{j}\right) \otimes\left(x_{j}, y_{j}, t_{j}\right)\right) \oplus\left(\alpha_{1}, \alpha_{2}, \alpha_{3}\right)}{\left(\sum_{j=1}^{n}\left(m_{j}, n_{j}, l_{j}\right) \otimes\left(x_{j}, y_{j}, t_{j}\right)\right) \oplus\left(\beta_{1}, \beta_{2}, \beta_{3}\right)}$

subject to $\sum_{j=1}^{n}\left(a_{i j}, b_{i j}, c_{i j}\right) \otimes\left(x_{j}, y_{j}, t_{j}\right)\{\preceq, \approx, \succeq\}\left(b_{i}, g_{i}, h_{i}\right)$, for all $i=1,2, \ldots, m, \quad\left(x_{j}, y_{j}, t_{j}\right) \succeq \tilde{0}$.

Now, since $\left(x_{j}, y_{j}, t_{j}\right)$ is a triangular fuzzy number, then

$$
x_{j} \leq y_{j} \leq t_{j}, \mathrm{j}=1,2, \ldots, \mathrm{m} .
$$

The relation (4.1) is called bounded constraints.

Now, using the arithmetic operations and partial ordering relations, we decompose the given FFLFP problem as follows:

Max. $z_{1}=$ Lower value of $\frac{\left(\sum_{j=1}^{n}\left(p_{j}, q_{j}, r_{j}\right) \otimes\left(x_{j}, y_{j}, t_{j}\right)\right) \oplus\left(\alpha_{1}, \alpha_{2}, \alpha_{3}\right)}{\left(\sum_{j=1}^{n}\left(m_{j}, n_{j}, l_{j}\right) \otimes\left(x_{j}, y_{j}, t_{j}\right)\right) \oplus\left(\beta_{1}, \beta_{2}, \beta_{3}\right)}$

Max. $z_{2}=$ Middle value of $\frac{\left(\sum_{j=1}^{n}\left(p_{j}, q_{j}, r_{j}\right) \otimes\left(x_{j}, y_{j}, t_{j}\right)\right) \oplus\left(\alpha_{1}, \alpha_{2}, \alpha_{3}\right)}{\left(\sum_{j=1}^{n}\left(m_{j}, n_{j}, l_{j}\right) \otimes\left(x_{j}, y_{j}, t_{j}\right)\right) \oplus\left(\beta_{1}, \beta_{2}, \beta_{3}\right)}$ 
Max. $z_{3}=$ Upper value of $\frac{\left(\sum_{j=1}^{n}\left(p_{j}, q_{j}, r_{j}\right) \otimes\left(x_{j}, y_{j}, t_{j}\right)\right) \oplus\left(\alpha_{1}, \alpha_{2}, \alpha_{3}\right)}{\left(\sum_{j=1}^{n}\left(m_{j}, n_{j}, l_{j}\right) \otimes\left(x_{j}, y_{j}, t_{j}\right)\right) \oplus\left(\beta_{1}, \beta_{2}, \beta_{3}\right)}$

subject to

$$
\begin{aligned}
& \sum_{j=1}^{n} \text { lower value of }\left(\left(a_{i j}, b_{i j}, c_{i j}\right) \otimes\left(x_{j}, y_{j}, t_{j}\right)\right)\{\leq,=, \geq\} b_{i}, \text { for all } i=1,2, \ldots, m ; \\
& \sum_{j=1}^{n} \text { middle value of }\left(\left(a_{i j}, b_{i j}, c_{i j}\right) \otimes\left(x_{j}, y_{j}, t_{j}\right)\right)\{\leq,=, \geq\} g_{i}, \text { for all } i=1,2, \ldots, m ; \\
& \sum_{j=1}^{n} \text { upper value of }\left(\left(a_{i j}, b_{i j}, c_{i j}\right) \otimes\left(x_{j}, y_{j}, t_{j}\right)\right)\{\leq,=, \geq\} h_{i}, \text { for all } i=1,2, \ldots, m
\end{aligned}
$$

and all decision variables are non-negative.

From the above decomposition problem, we construct the following three crisp LFP problems namely, middle level problem $(M L P)$, upper level problem $(U L P)$ and lower level problem $(L L P)$ as follows:

$$
(M L P) \text { Max. } z_{2}=\text { Middle value of } \frac{\left(\sum_{j=1}^{n}\left(p_{j}, q_{j}, r_{j}\right) \otimes\left(x_{j}, y_{j}, t_{j}\right)\right) \oplus\left(\alpha_{1}, \alpha_{2}, \alpha_{3}\right)}{\left(\sum_{j=1}^{n}\left(m_{j}, n_{j}, l_{j}\right) \otimes\left(x_{j}, y_{j}, t_{j}\right)\right) \oplus\left(\beta_{1}, \beta_{2}, \beta_{3}\right)}
$$

subject to constraints in the decomposition problem in which at least one decision variable of the $(M L P)$ occurs and all decision variables are non-negative.

$$
(U L P) \text { Max. } \quad z_{3}=\text { Upper value of } \frac{\left(\sum_{j=1}^{n}\left(p_{j}, q_{j}, r_{j}\right) \otimes\left(x_{j}, y_{j}, t_{j}\right)\right) \oplus\left(\alpha_{1}, \alpha_{2}, \alpha_{3}\right)}{\left(\sum_{j=1}^{n}\left(m_{j}, n_{j}, l_{j}\right) \otimes\left(x_{j}, y_{j}, t_{j}\right)\right) \oplus\left(\beta_{1}, \beta_{2}, \beta_{3}\right)}
$$

subject to $z_{3} \geq z_{2}^{\circ}$;

constraints in the decomposition problem in which at least one decision variable of the $(U L P)$ occurs and are not used in $(M L P)$;

all variables in the constraints and objective function in $(U L P)$ must satisfy the bounded constraints;

replacing all values of the decision variables which are obtained in $(M L P)$ and all decision variables are non-negative.

And

$$
\text { (LLP) Max. } z_{1}=\text { Lower value of } \frac{\left(\sum_{j=1}^{n}\left(p_{j}, q_{j}, r_{j}\right) \otimes\left(x_{j}, y_{j}, t_{j}\right)\right) \oplus\left(\alpha_{1}, \alpha_{2}, \alpha_{3}\right)}{\left(\sum_{j=1}^{n}\left(m_{j}, n_{j}, l_{j}\right) \otimes\left(x_{j}, y_{j}, t_{j}\right)\right) \oplus\left(\beta_{1}, \beta_{2}, \beta_{3}\right)}
$$

subject to $z_{1} \leq z_{2}^{\circ}$;

constraints in the decomposition constraints in which at least one decision variable of the $(L L P)$ occurs which are not used in $(M L P)$ and $(U L P)$;

all variables in the constraints and objective function in $(L L P)$ must satisfy the bounded constraints;

replacing all values of the decision variables which are obtained in the $(M L P)$ and $(U L P)$ and all decision variables are non-negative, where $z_{2}^{\circ}$ is the optimal objective value of $(M L P)$.

Now, we prove the following theorem which is used in the proposed method to solve the FFLFP problem.

Theorem 4.1 Let $\left[x_{M}^{\circ}\right]=\left\{x_{j}^{\circ}, x_{j}^{\circ} \in M\right\}$ be an optimal solution of $(M L P),\left[x_{U}^{\circ}\right]=\left\{x_{j}^{\circ}, x_{j}^{\circ} \in U\right\}$ be an optimal solution of $(U L P)$ and $\left[x_{L}^{\circ}\right]=\left\{x_{j}^{\circ}, x_{j}^{\circ} \in L\right\}$ be an optimal solution of $(L L P)$ where $L, M$ and $U$ are sets of 
decision variables in the $(L L P),(M L P)$ and $(U L P)$ respectively. Then $\left\{\tilde{x}_{j}^{\circ}=\left(x_{j}^{1}, x_{j}^{2}, x_{j}^{3}\right), j=1,2, \ldots, n\right\}$ is an optimal fuzzy solution to the given problem (FP) where each one of $x_{j}^{\circ 1}, x_{j}^{\circ 2}$ and $x_{j}^{\circ 3}, j=1,2, \ldots, n$ is an element of $L, M$ and $U$.

Proof: Let $\left[\tilde{y}_{j}\right]=\left\{\tilde{y}_{j}, j=1,2, \ldots, n\right\}$ be a feasible solution of (FP). Clearly, $\left[y_{M}\right],\left[y_{U}\right]$ and $\left[y_{L}\right]$ are feasible solutions of $(M L P),(U L P)$ and $(L L P)$ respectively.

Now, since $\left[x_{M}^{\circ}\right],\left[x_{U}^{\circ}\right]$ and $\left[x_{L}^{\circ}\right]$ are optimal solutions of $(M L P),(U L P)$ and $(L L P)$ respectively, we have $Z_{1}\left(\left[x_{L}^{\circ}\right]\right) \geq Z_{1}\left(\left[y_{L}\right]\right) ; Z_{2}\left(\left[x_{M}^{\circ}\right]\right) \geq Z_{2}\left(\left[y_{M}\right]\right)$ and $Z_{3}\left(\left[x_{U}^{\circ}\right]\right) \geq Z_{3}\left(\left[y_{U}\right]\right)$.

This implies that $Z\left(\left[\tilde{x}_{j}^{\circ}\right]\right) \geq Z\left(\left[\tilde{y}_{j}\right]\right)$, for all feasible solution of the problem $(\mathrm{P})$.

Therefore, $\left\{\tilde{x}_{j}^{\circ} \approx\left(x_{j}^{\circ 1}, x_{j}^{\circ 2}, x_{j}^{\circ 3}\right), j=1,2, \ldots, n\right\}$ is an optimal fuzzy solution to the given problem (FP) where each one of $x_{j}^{\circ 1}, x_{j}^{\circ 2}$ and $x_{j}^{\circ 3}, j=1,2, \ldots, n$ is an element of $L, M$ and $U$.

Hence the theorem is proved.

Remark 4.1 In the case of LFP problem involving trapezoidal fuzzy numbers and variables, we decompose it into four crisp LFP problems and then, we solve the middle level problems (second and third problems) first. Then, we solve the upper level and lower level problems and then, we obtain an optimal fuzzy solution to the given LFP problem involving trapezoidal fuzzy numbers and variables.

Remark 4.2 In the case of LFP problem involving interval fuzzy numbers and/or interval variables, we decompose it into two crisp LP problems and then, we solve the upper level problem first. Then, we solve the lower level problem and then, we attain the interval optimal solution to the given LFP problem involving interval numbers and / or interval variables.

Now, we introduce a new method namely, decomposition-restriction method to the FFLFP problem (FP) for obtaining an optimal fuzzy solution, based on the decomposition principle and the denominator objective restriction method.

The proposed method proceeds as follows:

Step 1: Construct three crisp LFP problems namely Middle level problem, Upper level problem and Lower level problem from the given FLFP problem.

Step 2: Solve the Middle level problem by the denominator objective restriction method.

Step 3: Using the results of Step 2 and the denominator objective restriction method, solve the Upper level problem.

Step 4: Using the results of Step 2, Step 3 and the denominator objective restriction method, solve the Lower level problem.

Step 5: Using the results of Step 2, Step 3 and Step 4, obtain an optimal fuzzy solution to the given FFLFP problem by the Theorem 4.1.

The proposed method is illustrated with the following example.

Example 4.1 Consider the following FFLFP problem:

$$
\text { Maximize } \tilde{Z}=\frac{(0,1,2) \tilde{x}_{1} \oplus(-2,-1,0) \tilde{x}_{2} \oplus(0,1,2)}{(0,1,2) \tilde{x}_{1} \oplus(0,1,2) \tilde{x}_{2} \oplus(1,2,3)}
$$

subject to $(0,1,2) \tilde{x}_{1}+(0,1,2) \tilde{x}_{2} \preceq(1,2,3), \quad(0,1,2) \tilde{x}_{1}+(-2,-1,0) \tilde{x}_{2} \preceq(0,1,2) ; \quad \tilde{x}_{1}, \tilde{x}_{2} \geq 0$.

Let $\tilde{x}_{1}=\left(x_{1}, y_{1}, t_{1}\right), \quad \tilde{x}_{2}=\left(x_{2}, y_{2}, t_{2}\right)$ and $\tilde{z}=\left(z_{1}, z_{2}, z_{3}\right)$.

Now, the decomposition problems of the given FFLFP problem are given below:

$$
\begin{gathered}
\text { Maximize } z_{1}=\frac{-2 t_{2}}{2 t_{1}+2 t_{2}+3} \\
\text { Maximize } z_{2}=\frac{y_{1}-y_{2}+1}{y_{1}+y_{2}+2} \\
\text { Maximize } z_{3}=2 t_{1}+2
\end{gathered}
$$


subject to $0 x_{1}+0 x_{2} \leq 1 ;-2 t_{2} \leq 0 ; y_{1}+y_{2} \leq 2 ; y_{1}-y_{2} \leq 1 ; 2 t_{1}+2 t_{2} \leq 3 ; 2 t_{1} \leq 2 ; x_{1}, x_{2} \geq 0, y_{1}, y_{2} \geq 0, t_{1}, t_{2} \geq 0$.

Now, the Middle Level problem is given below:

$$
\text { (MLP) Maximize } z_{2}=\frac{y_{1}-y_{2}+1}{y_{1}+y_{2}+2}
$$

subject to $y_{1}+2 y_{2} \leq 2 ; y_{1}-y_{2} \leq 1 ; y_{1}, y_{2} \geq 0$.

Now, solving the problem (MLP) by the denominator objective restriction method, we attain an optimal solution $y_{1}=1 ; y_{2}=0$ and $z_{2}=\frac{2}{3}$.

Now, the Upper Level problem is given below:

$$
\text { (ULP) Maximize } z_{3}=2 t_{1}+2
$$

subject to $2 t_{1}+2 \geq \frac{2}{3} ; 2 t_{1}+2 t_{2} \leq 3 ; t_{1} \leq 1 ; t_{1} \geq y_{1} ; t_{2} \geq y_{2} ; t_{1}, t_{2} \geq 0$.

Now, solving the problem (ULP) with $y_{1}=1 ; y_{2}=0$ using the denominator objective restriction method, he optimal solution to the problem (ULP) is $t_{1}=1 ; t_{2}=0$ and $z_{3}=4$.

Now, the Lower Level problem:

$$
\text { (LLP) Maximize } z_{1}=\frac{-2 t_{2}}{2 t_{1}+2 t_{2}+3}
$$

subject to $\frac{-2 t_{2}}{2 t_{1}+2 t_{2}+3} \leq \frac{2}{3} ; 2 t_{1}+2 t_{2} \leq 3 ; t_{1} \leq 1 ; t_{1} \geq y_{1} ; t_{2} \geq y_{2} ; t_{1}, t_{2} \geq 0$.

Now, substituting $t_{1}=1$ and $t_{2}=0$ in the problem (LLP), the optimal solution is $t_{1}=1, t_{2}=0$ and $z_{1}=0$.

Now, since $x_{1} \leq y_{1}, x_{2} \leq y_{2}$ and $x_{1}, x_{2} \geq 0$, we can conclude that $x_{2}=0$ and $x_{1}=x_{0}$ where $x_{0} \in[0,1]$.

Therefore, an optimal fuzzy solution to the given FFLFP problem is $\tilde{x}_{1} \approx\left(x_{0}, 1,1\right), \tilde{x}_{2} \approx(0,0,0)$ and Max. $\tilde{z} \approx\left(0, \frac{2}{3}, 4\right)$ where $x_{0} \in[0,1]$.

Remark 4.3 In Bogdana Stanojevi'ca and Stancu-Minasianb (2012), the optimal fuzzy solution to the FFLFP problem (Example 4.1.) is $\tilde{x}_{1} \approx(0,1,1), \quad \tilde{x}_{2} \approx(0,0,0)$ and the maximum value of $\tilde{z} \approx(0,0.55,1.09)$ by a new method which is based on Charnes-Copper method and multiobjective nonlinear programming, but by the decomposition-restriction method, the optimal fuzzy solution to the same FFLFP problem is $\tilde{x}_{1} \approx\left(x_{0}, 1,1\right)$, $\tilde{x}_{2} \approx(0,0,0)$ and the maximum value $\tilde{z} \approx\left(0, \frac{2}{3}, 4\right)$ where $x_{0} \in[0,1]$.

\section{Conclusion}

In this paper, the denominator objective restriction method is developed for solving LFP problems based only on simplex method in. It is easy to understand, compute and also, to interpret. In the second part of the proposed method, that is, solving the problem (D), each iteration solution is a next level accepted solution to the LFP problem that may be used by decision makers according to their situations if they need. Further, the decomposition-restriction method is used to solve the FFLFP problem in which the fuzzy ranking function, transformation technique and multi-objective non-linear programming technique are not used, but it is based on the decomposition principle and the denominator objective restriction method. Both the methods can serve decision makers by providing an appropriate best solution to a variety of linear fractional programming models having crisp or fuzzy parameters and variables in a simple and effective manner.

\section{Acknowledgements}

We owe a lot to Dr. G. Natarajan and Prof. M.A. Mohamed Sahul Hameed, VIT University, Vellore in reviewing our paper which helped us to complete this work.

\section{References}

Bajalinov, E. B. (2003). Linear-fractional-Programming Theory, Methods, Applications and Software. Boston: Kluwer Academic publishers. 
Bellman, R. E., \& Zadeh, L. A. (1970). Decision Making in a Fuzzy Environment. Management Science, 17, 141-164. http://dx.doi.org/10.1287/mnsc.17.4.B141

Bitran, G. R., \& Novaes, A. J. (1973). Linear programming with a fractional objective function. Journal of Operations Research, 21, 22-29. http://dx.doi.org/10.1287/opre.21.1.22

Buckley, J. J., \& Feuring, T. (2000). Evolutionary Algorithm solution to fuzzy problems: fuzzy linear programming. Fuzzy Sets and Systems, 109, 35-53. http://dx.doi.org/10.1016/S0165-0114(98)00022-0

Charnes, A., \& Cooper, W. W. (1962). Programming with linear functional. Naval Research Logistics Quarterly, 9, 181-186. http://dx.doi.org/10.1002/nav.3800090303

Dantzig, G. B. (1962). Linear Programming and extension. Princeton University Press, Princeton, New Jersey.

Gilmore, P. C., \& Gomory, R. E. (1963). Linear programming approach to the cutting stock problem-Part 2. Operations Research, 11, 863-867. http://dx.doi.org/10.1287/opre.11.6.863

Hashemi, S. M., Modarres, M., Nasrabadi, E., \& Nasrabadi, M. M. (2006). Fully fuzzified linear programming, solution and duality. Journal of Intelligent and Fuzzy Systems, 17, 253-261.

Isbell, J. R., \& Marlow, W. H. (1956). Attrition games. Naval Research Logistics Quarterly, 3, 1-99. http://dx.doi.org/10.1002/nav.3800030108

Jayalakshmi, M., \& Pandian, P. (2012). A New Method for Finding an Optimal Fuzzy Solution Fully Fuzzy Linear Programming Problems. International Journal of Engineering Research and Applications, 2, 247-254.

Li, D. F., \& Chen, S. (1996). A fuzzy programming approach to fuzzy linear fractional programming with fuzzy coefficients. The Journal of Fuzzy Mathematics, 4, 829-834.

Martos, B. (1964). Hyperbolic programming. Naval Research Logistics Quarterly, 11, $135-155$. http://dx.doi.org/10.1002/nav.3800110204

Mikaeilvand, N., Allahviranloo, T., Hosseinzadeh, F., \& Khorasany Kiasary, M. (2008). Fully fuzzy linear programming problem with positive or negative core. Far East Journal of Applied Mathematics, 33, 337-350.

Mojtaba, B., Azmin, S. R., \& Mansour, S. (2012). Solving linear fractional programming problems with interval coefficients in the objective function-A new approach. Applied Mathematical Sciences, 6, 3442-3452.

Nachammai, Al., \& Thangaraj, P. (2012). Solving fuzzy linear fractional programming problem using metric distance ranking. Applied Mathematical Sciences, 6, 1275-1285.

Odior, A. O. (2012). An approach for solving linear fractional programming problems. International Journal of Engineering and Technology, 1, 298-304.

Pandey, P., \& Punnen, A. P. (2007). A simplex algorithm for piecewise-linear fractional programming problems. European Journal of Operational Research, 178, 343-358. http://dx.doi.org/10.1016/j.ejor.2006.02.021

Pop, B., \& Stancu-Minasian, I. M. (2008). A method of solving fully fuzzified fractional programming problems. J. Appl. Math. Comput., 27, 227-242. http://dx.doi.org/10.1007/s12190-008-0052-5

Schaible, S. (1981). Fractional programming: Applications and Algorithms. European Journal of Operational Research, 7, 111-120. http://dx.doi.org/10.1016/0377-2217(81)90272-1

Sharma, J. K., Gupta, A. K., \& Gupta, M. P. (1980). Extension of simplex technique for solving fractional programming problems. Indian Journal of Pure and Applied Mathematics, 11, 961-968.

Stancu-Minasian, I. M. (1997). Fractional programming: Theory, methods and applications. Kluwer Academic Publishers, Dordrecht. http://dx.doi.org/10.1007/978-94-009-0035-6

Stancu-Minasian, I. M. (2006). A sixth bibliography of fractional programming. Optimization, 55, $405-428$. http://dx.doi.org/10.1080/02331930600819613

Stanojevi'c, B., \& Stancu-Minasian, I. M. (2012). Evaluating fuzzy inequalities and solving fully fuzzified linear fractional program. Yugoslav Journal of Operations Research, 22, 41-50. http://dx.doi.org/10.2298/YJOR110522001S

Swarup, K. (1965). Linear fractional functional programming. Operation Research, 13, 1029-1036. http://dx.doi.org/10.1287/opre.13.6.1029 
Tanaka, H., Okuda, T., \& Asai, K. (1973). On Fuzzy Mathematical Programming. Journal of Cybernetics and Systems, 3, 37-46. http://dx.doi.org/10.1080/01969727308545912

Tantawy, S. F. (2007). Using feasible directions to solve linear fractional programming problems. Australian Journal of Basic and Applied Sciences, 1, 109-114.

Tantawy, S. F. (2008). A new procedure for solving linear fractional programming problems. Mathematical and Computer Modeling, 48, 969-973. http://dx.doi.org/10.1016/j.mcm.2007.12.007

Wagner, H. M., \& Yuan, J. S. C. (1968). Algorithm equivalence in linear fractional programming. Management Science, 14, 301-306. http://dx.doi.org/10.1287/mnsc.14.5.301 